\title{
The Method of Oilfield Development Risk Forecasting and Early Warning Using Revised Bayesian Network
}

\author{
Yihua Zhong, Yuxin Liu, Xuxu Lin, and Shiming Luo \\ School of Science, Southwest Petroleum University, Chengdu, Sichuan 610500, China \\ Correspondence should be addressed to Yihua Zhong; zhongyh_65@126.com
}

Received 26 November 2015; Revised 14 January 2016; Accepted 27 January 2016

Academic Editor: Egidijus R. Vaidogas

Copyright ( 2016 Yihua Zhong et al. This is an open access article distributed under the Creative Commons Attribution License, which permits unrestricted use, distribution, and reproduction in any medium, provided the original work is properly cited.

\begin{abstract}
Oilfield development aiming at crude oil production is an extremely complex process, which involves many uncertain risk factors affecting oil output. Thus, risk prediction and early warning about oilfield development may insure operating and managing oilfields efficiently to meet the oil production plan of the country and sustainable development of oilfields. However, scholars and practitioners in the all world are seldom concerned with the risk problem of oilfield block development. The early warning index system of blocks development which includes the monitoring index and planning index was refined and formulated on the basis of researching and analyzing the theory of risk forecasting and early warning as well as the oilfield development. Based on the indexes of warning situation predicted by neural network, the method dividing the interval of warning degrees was presented by " $3 \sigma$ " rule; and a new method about forecasting and early warning of risk was proposed by introducing neural network to Bayesian networks. Case study shows that the results obtained in this paper are right and helpful to the management of oilfield development risk.
\end{abstract}

\section{Introduction}

Because the oilfield development has the basic characteristics of high risk and high investment as well as complicated process, it is very important to study oilfield development risk forecasting and early warning in order to keep the oil production safe and reduce decision-making mistakes to be caused by risk. Up to now, many scholars have studied the problems on safety related risk and risk-based decisionmaking [1-17]. For example, Khan et al. summarized and reviewed methods and models on process safety and risk management in recent years; moreover, they figured out their present research trends and future research direction which includes dynamic risk assessment and management as well as advanced consequence modeling [1]. Khan et al. pointed out challenges on development of natural resources in harsh environments [2]. Because Bayesian network (BN) is currently being used for applications related to safety and risk assessment, we first highlight those works that were solved by using Bayesian network (BN) and its improved methods in different industries as follows. Musharraf et al. presented a data collection methodology using a virtual environment for a simplified BN model of offshore emergency evacuation and the probability of failure in the offshore emergency evacuation calculated by BN model aiming at human reliability analysis [3]. Khakzad et al. demonstrated the application of bow-tie and $\mathrm{BN}$ methods in conducting quantitative risk analysis of drilling operations and verified BN method which may provide a better vision of well control safety issues and greater value than the bow-tie model [4]. Abimbola et al. presented a risk assessment methodology based on BN for analyzing the safety critical components and consequences of possible pressure regimes in constant bottom-hole pressure techniques of managed pressure drilling [5]. Vinnem et al. developed a dynamic risk analysis methodology that focuses on translating the blowout flowchart directly into $\mathrm{BN}$ and showed that $\mathrm{BN}$ is an effective technique, which can be used in risk analysis and failure prediction for the offshore industry [6]. Bhandari et al. proposed applying BN to conduct a dynamic safety analysis of deepwater managed pressure drilling operations (MPD) and underbalanced drilling (UBD) operations [7]. Khakzad et al. illustrated how BN helps to overcome the limitations of bow-tie (BT) method; and pointed out that BN can be used in dynamic safety analysis of a wide range of accident scenarios [8]. Yu et al. proposed a two-stage fault diagnosis 
technique for process operations [9], in which the modified independent component analysis is used in the first stage for fault detection and to identify the faulty monitored variable, and $\mathrm{BN}$ is in the second stage with considering the process variables and their dependence obtained from the process flow diagram. Yuan et al. proposed a methodology for risk analysis of dust explosion scenarios based on BN and a bowtie diagram [10]. Khakzad presented a methodology based on dynamic BN to model both the spatial and the temporal evolutions of domino effects and also to quantify the most probable sequence of accidents in a potential domino effect with having significance of foreseeing the temporal evolution of domino effects and predicting the most probable sequence of accidents on a domino effect in the allocation of preventive and protective safety measure [11]. Khakzad et al. developed a methodology based on event tree and hierarchical Bayesian analysis to establish informative distributions for offshore blowouts using data of near accidents and implemented the methodology in a Markov Chain Monte Carlo framework and applied it to risk assessment of offshore blowouts in the Gulf of Mexico [12]. Baksh et al. improved the shortcomings of the existing accident model to propose predictive accident modeling methodologies through mitigating restrictive sequential progression assumption and using BN approach [13]. Yang et al. performed a similar analysis as Kalantarnia et al. (2009) [14] to reduce the uncertainty of fault tree calculation using Bayes' theorem [15]. Besides above methods, some scholars also developed other research methods. For example, Alaneme and Igboanugo provided a veritable tool that systematically transforms the qualitative risk variables from its linguistic expressions to quantitative functions using fuzzy logic in combination with conventional risk analysis techniques with marginal oilfield risks [16]. Lavasani et al. proposed a fuzzy logic based risk assessment methodology to perform quantitative risk assessment for offshore oil and gas wells [17].

The above works mainly used BN combined with fault tree and event tree and other methods to solve the analysis and assessment as well as diagnostics on risk. These revised Bayesian networks may provide a convincing basis for BN structure learning and parameter learning from another aspect. The concept of integrating neural network with BN has been present for a long time [18]. Works on this concept include two fields: one is to deal with the problems related to $\mathrm{NN}$ from Bayesian perspective [18-22]; the other is to deal with the problems related to $\mathrm{BN}$ from $\mathrm{NN}$ perspective. However, the latter is seldom studied. As an example, Maschio and Schiozer proposed replacing the flow simulator by proxy models generated by artificial neural network (ANN) to formulate the posterior distribution of Bayesian inference in the history matching [23]. Based on the ability for NN to be known as $100 \%$ fitting any function, we propose introducing $\mathrm{NN}$ to $\mathrm{BN}$ to calculate the posterior probability distribution of $\mathrm{BN}$ node under the evidence.

In a word, the literature investigation showed that there are a few studies focusing on safety related risk or risk-based decision-making of petroleum industry, and they mainly concentrated on risk analysis, risk assessment, and prediction in microscopic aspects such as drilling and blowout, while the researches in the macro-aspect are almost focused on the monitoring, predicting, and early warning of operation risk about oilfield enterprise [24-26]. However, they are seldom concerned with risk problem of oilfield block development from the point of microcosmic perspective and control.

This paper aims to forecast and early warn of oilfield block development risk to study risk management in the oilfield development and its related issues, including theory of risk forecast and early warning, model and method of forecast, and early warning oilfield development risk which may provide the technique support for controlling risk by using the theory of oilfield development, forecasting warning, Bayesian networks, neural network and so forth, as well as case study to test the correctness of model and method presented in this paper. Particularly, we extract five blocks' development index based on the law of single well producing oil and transform them successfully into risk nodes using " $3 \sigma$ " rule. Then, we predict oil production by neural network; subsequently, those values were used for decision-making whether they are in the normal interval of yield or not. If one of them is not in the normal interval of yield, then we diagnose what makes prediction result of yield is abnormal by using $\mathrm{BN}$ and trace the source of risk.

\section{Theoretical Studies of Risk Predicting and Early Warning}

The risk is the root or state that makes events be in danger or cause loss; its theories of prediction and early warning include concepts and methods of risk identification, risk assessment, prediction, and early warning [27].

The base of forecasting and early warning is to identify risk and risk source. The risk sources are generally divided into two categories, the first category is innate existence and is not controlled by human; the second category is arising under the influence of people or things $[28,29]$.

In the study of forecasting and early warning of oilfield development risk, we classified all the indicators in three categories that are the index of warning situation, the index of warning sign, and the index of warning source. Indexes of warning situation are the events that are most taken care of in oilfield development, such as oil production and liquid production; indexes of warning sign are the indexes in contact with the indexes of warning situation and they are easily monitored. Indexes of warning source are causes of the risk. The key of risk prediction and early warning is measuring risk, but now there is no appropriate method to measure risk of oilfield development. At present, the main methods of risk prediction and early warning are neural network method [30], Monte Carlo method [31-33], fuzzy comprehensive evaluation method $[34,35]$, and Bayesian network method [36, 37]. In order to study the problem of oilfield development risk forecasting and early warning, we will present an improved Bayesian network method by introducing neural network to the structure learning of BN in this paper. 


\section{Research of Prediction and Early Warning on Oilfield Development Risk}

3.1. Early Warning Index System of Oilfield Development Risk. Since the process of oilfield development is very complex, it is quite difficult to identify, evaluate, predict, and early warn of the risks implied in this process. After studying reservoir physical and geological exploration, we adopt the thinking mode proposed in the book named Early Warning Dynamic System in the Oilfield Development [38] to construct the early warning index system of oilfield development risk, that is, first analyzing the association relationship among the testing indexes of single well to select testing index with larger association degree, then explaining these test indexes of single well to layer index, and finally refining the index system of oilfield block development risk. This index system contains oil production (OPRO), liquid production (LPRO), oil producing capacity (OPC), liquid producing capacity (LPC), water absorbing capacity (WAC), percolating capacity (PEC), and pressure maintaining level (PML). Among them, oil production and liquid production are the indexes of warning situation; other indexes are the indexes of warning sign [39].

\subsection{Method to Forecast Oilfield Development Risk}

3.2.1. Neural Network Prediction of Warning Situation Indexes. Neural network is abstracting and modeling of the human brain or biological neural network, which is connected by a large number of neurons. The artificial neuron is a simple mapping model; artificial neuron model can be expressed as

$$
\begin{aligned}
& S=\sum_{i=1}^{n} \omega_{i} x_{i}-\theta, \\
& y=\sigma(s),
\end{aligned}
$$

where $x_{i}(i=1,2, \ldots, n)$ is the input signal added to the input; $\omega_{i}$ is the weight coefficient of corresponding connection which is a coefficient to simulate the strength of synaptic transmission; $\sum$ shows the summation of the postsynaptic potential pulse signal; $\theta$ is the critical value of neurons; $\sigma(s)$ is the excitation function of artificial neurons, which is commonly expressed as one of two $S$ (Sigmoid) functions as follows $[40,41]$ :

$$
\begin{aligned}
& \sigma(s)=\frac{1}{1+e^{-1}}, \\
& \sigma(s)=\tanh (\beta s) .
\end{aligned}
$$

A lot of the same-form artificial neurons are arranged in layers to form neural network. The neurons receiving signal are called the input layer, the neurons of output signal are called the output layer, and the neurons not directly involved in the input or output are called the intermediate layer or hidden layer.

Input of neural network used in the prediction of oilfield development risk is warning sign indexes (five-block indexes); its output is warning situation indexes (two production indexes). Parameter $\omega_{i}$ of the network was trained

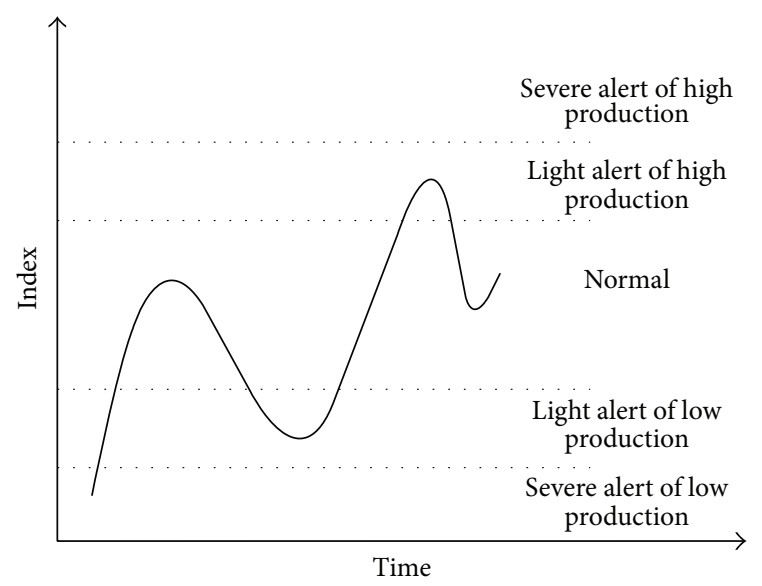

FIGURE 1: Interval of warning degree.

by historical data to be determined. This paper trained 1367 networks to select the best model as its predictive model. Its fitting precision is up to 0.002 , and the prediction accuracy reaches 0.08 .

3.2.2. Method to Determinate the Degree of Oilfield Development Risk. After predicting indexes of warning situation by neural network trained model, the interval of warning degree is determined by " $3 \sigma$ " rule, where $\sigma$ is the standard deviation which means the discrete degree of data in statistics. According to the statistical principle, if the value of sample deviating from its mathematical expectation $\mu$ is more than 1 time the standard deviation, then probability is only $31.74 \%$; if its value is more than 2 times the standard deviation, then probability is only $5 \%$; if its value is more than 3 times the standard deviation, then probability is less than $1 \%$, which is usually said to be the small probability event. Therefore, we may determine the degree that indexes of warning situation happen to risk according to the interval that sample value belongs to. The steps of this method are as follows.

Step 1. Calculate the expected value $\mu$ and standard deviation $\sigma$ of indexes of warning situation by their historic data.

Step 2. Divide the interval of warning degree into the normal interval $[\mu-\sigma, \mu+\sigma]$, the light abnormal intervals $[\mu-2 \sigma, \mu-\sigma]$ and $[\mu+\sigma, \mu+2 \sigma]$, and the severe abnormal intervals $(-\infty, \mu-$ $2 \sigma]$ and $[\mu+2 \sigma,+\infty)$ by " $3 \sigma$ " rule [42]; see Figure 1 .

Step 3. Predict indexes of warning situation by neural network trained model.

Step 4. Determine the warning degree on the indexes of warning situation. If the prediction value of index of warning situation falls in the normal interval, then it has no risk; if the prediction value of index of warning situation falls in the light abnormal interval, then it has lesser risk; if the prediction value of index of warning situation falls in the severe abnormal interval, then it has bigger risk. 


\subsection{The Early Warning Method of Oilfield Development Risk by Revised Bayesian Network}

\subsubsection{Bayesian Network Model of Early Warning}

(1) Structure Learning. Bayesian network is a structure to connect the nodes of causal relationship, which is usually determined by two kinds of methods. One is the manual learning method where people use experience to determine whether there is the directed edge connecting nodes or not; the other is machine learning method based on data driven learning, which uses $\mathrm{K} 2$ algorithm to search gradually to meet the conditions and score the highest network. The steps of machine learning are as follows.

Step 1. Define a random variable $S^{h}$.

Step 2. Determine its prior probability distribution $p\left(S^{h}\right)$.

Step 3. Calculate the posterior probability distribution $p\left(S^{h} \mid\right.$ $D)$; that is,

$$
p\left(S^{h} \mid D\right)=\frac{p\left(S^{h}, D\right)}{p(D)}=\frac{p\left(S^{h}\right) p\left(D \mid S^{h}\right)}{p(D)}
$$

where $p(D)$ is a constant which is not related with the structure; $p\left(D \mid S^{h}\right)$ is the likelihood function of boundary; it is calculated by [43]

$$
p\left(D \mid S^{h}\right)=\prod_{i=1}^{n} \prod_{j=1}^{q_{i}} \frac{\Gamma\left(\partial_{i j}\right)}{\Gamma\left(\partial_{i j}+N_{i j}\right)} \prod_{k=1}^{r_{i}} \frac{\Gamma\left(\partial_{i j k}+N_{i j k}\right)}{\Gamma\left(\partial_{i j k}\right)} .
$$

(2) Parameter Learning. The goal of parameter learning is to update the prior distribution of network variables. Let the joint probability distribution of variables $X=\left\{x_{1}, x_{2}, \ldots, x_{n}\right\}$ be stored in network $S$ (see [44]):

$$
p\left(x \mid \theta_{s}, S^{h}\right)=\prod_{i=1}^{n} p\left(x_{i} \mid p a_{i}, \theta_{i}, S^{h}\right)
$$

where $S^{h}$ means that the joint distribution can be decomposed according to $S ; D=\left\{X_{1}, X_{2}, \ldots, X_{n}\right\}$ is the random sample of the joint probability distribution of $X ; \theta_{s}$ is parameters variable of network, whose vector $\theta_{i}$ represents its uncertainty; $p\left(\theta_{S}, S^{h}\right)$ is a given prior probability density function. Thus, the learning problem of Bayesian network parameters can be expressed as calculating posterior distribution $p\left(\theta_{S} \mid D, S^{h}\right)$ after giving a random sample $D$ [45].

(3) Bayesian Network Inference. The essence of Bayesian network inference is to calculate the conditional probability distribution. Let the set of evidence variable be $E$ and let the set of query variable be $Q$; then, the Bayesian network inference is to calculate the posterior probability distribution of the query variable $Q$ in $E=e$, which is formulated as follows:

$$
\begin{aligned}
P(Q \mid E=e) & =\sum_{X-E} P\left(x_{1}, x_{2}, \ldots, x_{n}\right) \\
& =\sum_{X-E} \prod_{i=1}^{n} P\left(x_{i} \mid P a_{i}\right) .
\end{aligned}
$$

3.3.2. The Method of Forecasting and Early Warning Based on Neural Network and Bayesian Network. Because warning sign index is called fault source in the Bayesian network diagnostic theory, it is also risk source. Generally, the key problem in early warning is to predict whether risk source fault occurs or not, thus warning sign indexes may be dealt with with Boolean variables. Combining the method of risk prediction using neural network in Section 3.2 with diagnostic and inference function of Bayesian network, we presented a new method of forecasting and early warning for oilfield development risk as follows.

Step 1. Construct early warning index system of oilfield development risk using the method in Section 3.1.

Step 2. Generate an appropriate neural network model of forecasting warning situation indexes, whose input is the historical sample data of five warning signs indicators; its output is the historical sample data of warning situation indexes.

Step 3. Predict the value of warning situation indexes by neural network trained model in Step 2.

Step 4. Determine the warning degree of predictive value about warning situation indexes by using the method in Section 3.2.2.

Step 5. Use the history data and the experience of experts to learn Bayesian network structure and parameter.

Step 6. Input the neural network prediction results into Bayesian networks and calculate posterior probability distribution under this evidence.

Step 7. Compare the posterior probability of fault and identify the source of the risk caused by warning situation indexes.

\section{Case Studies}

In this section, we will analyze and validate that the predictive models established and the new methods of early warning proposed above are correct and feasible by the historical data of oilfield block development in Table 1.

4.1. The Predicting of Warning Situation Index (Production) on Oilfield Development Risk. According to the methods in Sections 3.1 and 3.2.1, we build a neural network model which takes the warning signs indexes (OPC, LPC, PEC, WAC, and $\mathrm{PML}$ ) as input and the warning situation indexes (OPRP, LPRO) as output to predict the warning situation index 
TABLE 1: The historical data of an oilfield from 2007.07 to 2009.12 .

\begin{tabular}{lccccccc}
\hline Date & OPRP & LPRO & LPC & OPC & PEC & WAC & PML \\
\hline 2007.07 & 5637 & 92297 & 0.3018 & 0.0075 & 1549.26 & 1.5842 & 5.1452 \\
2007.08 & 5248 & 92695 & 0.3016 & 0.0072 & 1629.54 & 1.6147 & 3.4864 \\
2007.09 & 4855 & 91884 & 0.3017 & 0.0074 & 1302.18 & 1.6174 \\
$\vdots$ & $\vdots$ & $\vdots$ & $\vdots$ & $\vdots$ & $\vdots$ & $\vdots$ & $\vdots$ \\
2009.10 & 4254 & 112409 & 0.3017 & 0.007 & 234.916 & 1.504 \\
2009.11 & 3439 & 98884 & 0.3018 & 0.0069 & 231.74 & 1.5106 \\
2009.12 & 3260 & 97499 & 0.3018 & 0.0069 & 226.067 & 1.5744 \\
\hline
\end{tabular}

TABLE 2: Comparison table of neural network predictive value and the actual value.

\begin{tabular}{lcccc}
\hline & \multicolumn{2}{c}{ Predictive value } & \multicolumn{2}{c}{ Actual value } \\
& Oil production $(\mathrm{t})$ & Liquid production $(\mathrm{t})$ & Oil production $(\mathrm{t})$ & Liquid production $(\mathrm{t})$ \\
\hline 2010.01 & 2330 & 99000 & 2552 & 98026 \\
2010.02 & 3060 & 105800 & 3398 & 97475 \\
2010.03 & 3690 & 109240 & 3705 & 117607 \\
\hline
\end{tabular}

(production) of the oilfield development risk from 2010.01 to 2010.03 based on the data in Table 1 . Their predictive values and actual values are shown in Table 2.

4.2. The Determining of Warning Degree on the Oilfield Development Risk. Firstly, we draw the trend figure of actual production change as Figure 2. This figure shows that the liquid production appears as upward trend during the whole period, but oil production appears as downward trend after 2007.07, which means that this oilfield development has entered the high water-cut period, which needs to be injected by a large amount of water to increase oil production after 2007.07. Then, we use the method in Section 3.2.2 to determine the interval of warning degree as in Table 3 and the warning degree of the predicted values on warning situation index as in Table 4.

\subsection{Early Warning for Oilfield Development Risk}

4.3.1. The Establishing of the Bayesian Network Model. We use genie software to construct the Bayesian network model for the index system of oilfield block development risk which contains OPRO, LPRO, OPC, LPC, PEC, WAC, and PML as in Figure 3 and learn the parameter of Bayesian network based on the history data and the experience of experts as in Figure 4. It shows that the probability of oil output occurring at low production risk is bigger according to its historical data. It is the reason that we do not use Bayesian network to forecast oilfield block development risk. If the value of five warning sign indexes is higher than the average value plus standard deviation or lower than the sum of average value and standard deviation, then we defined its state as fault. Otherwise, its state is normal.

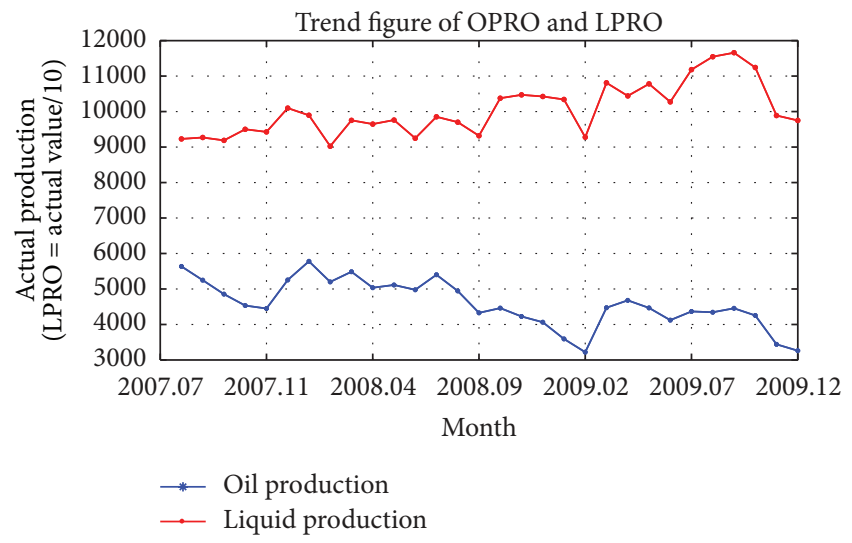

FIgURE 2: Trend of actual production change.

4.3.2. The Inference of Bayesian Network. We shall take the prediction results of neural network in Section 4.1 as observational evidence and use the inference of Bayesian network to get warning signs indexes which is the most risky under this evidence. That is to input the forecast results into the Bayesian networks and calculate posterior probability distribution of the warning signs indexes to find the origin of the risks. Three group evidences and their inference results are as in Figures 5-7.

Figure 5 shows that the most likely risk point which causes such evidence is LPC, for the posterior probability of LPC fault is much larger than others, which is $76 \%$. Reasoning results are matching with the actual situation.

Figure 6 shows that the most likely risk point which causes such evidence is PEC, for the posterior probabilities 
TABLE 3: The interval of warning degree based on $3 \sigma$ rule.

\begin{tabular}{lcccc}
\hline Warning level & Warning degree & \multicolumn{2}{c}{ Oil production } & \multicolumn{2}{c}{ Liquid production } \\
\hline Low production heavy warning (Lhw) & 1 & 0 & 3249 & 0 \\
Low production light warning (Llw) & 2 & 3249 & 3919 & 85825 \\
Normal (Nor) & 3 & 3919 & 5259 & 93142 \\
High production light warning (Hlw) & 4 & 5259 & 5929 & 107776 \\
High production heavy warning (Hhw) & 5 & 5929 & $+\infty$ & 1152 \\
\hline
\end{tabular}

TABLE 4: The predicted values of productions and the warning degree of them.

\begin{tabular}{lcccc}
\hline & Oil production $(\mathrm{t})$ & Warning degree & Liquid production $(\mathrm{t})$ & Warning degree \\
\hline 2010.01 & 2330 & 1 & 99000 & 3 \\
2010.02 & 3260 & 2 & 105800 & 3 \\
2010.03 & 3690 & 2 & 109240 & 4 \\
\hline
\end{tabular}

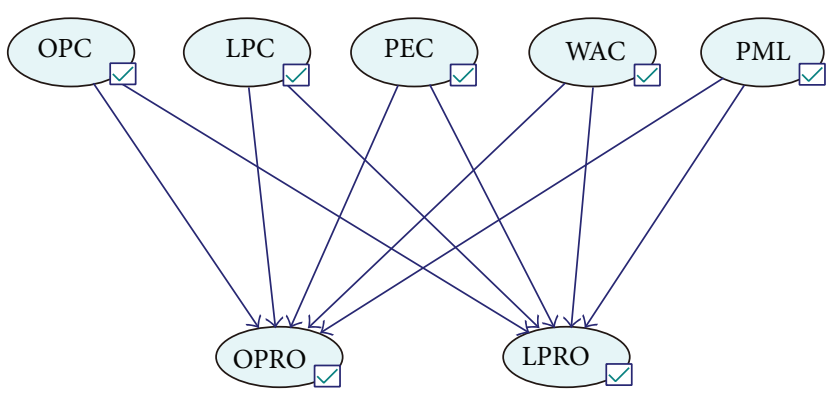

FIGURE 3: The Bayesian network construction model of oilfield block development risk.

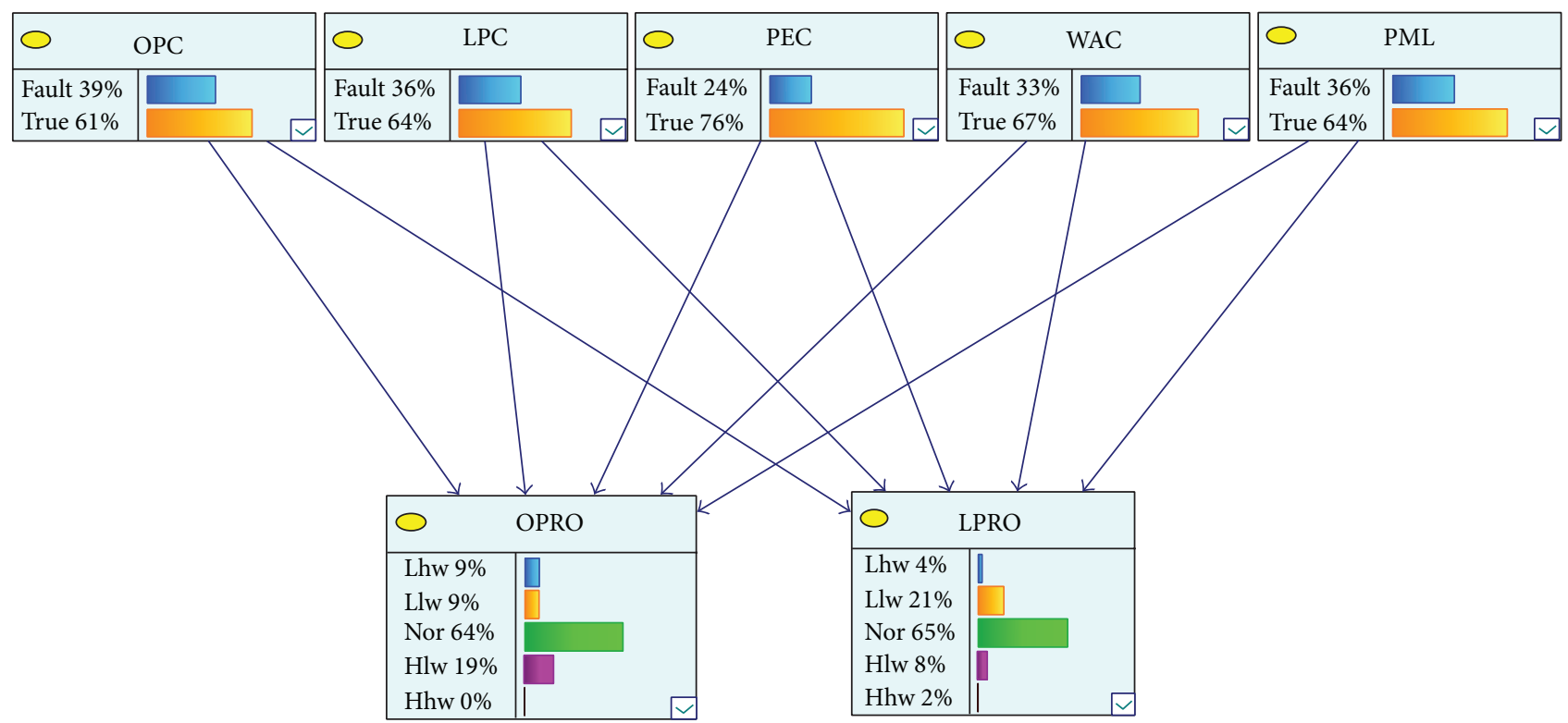

FIgURE 4: The parameter learning of Bayesian network. 


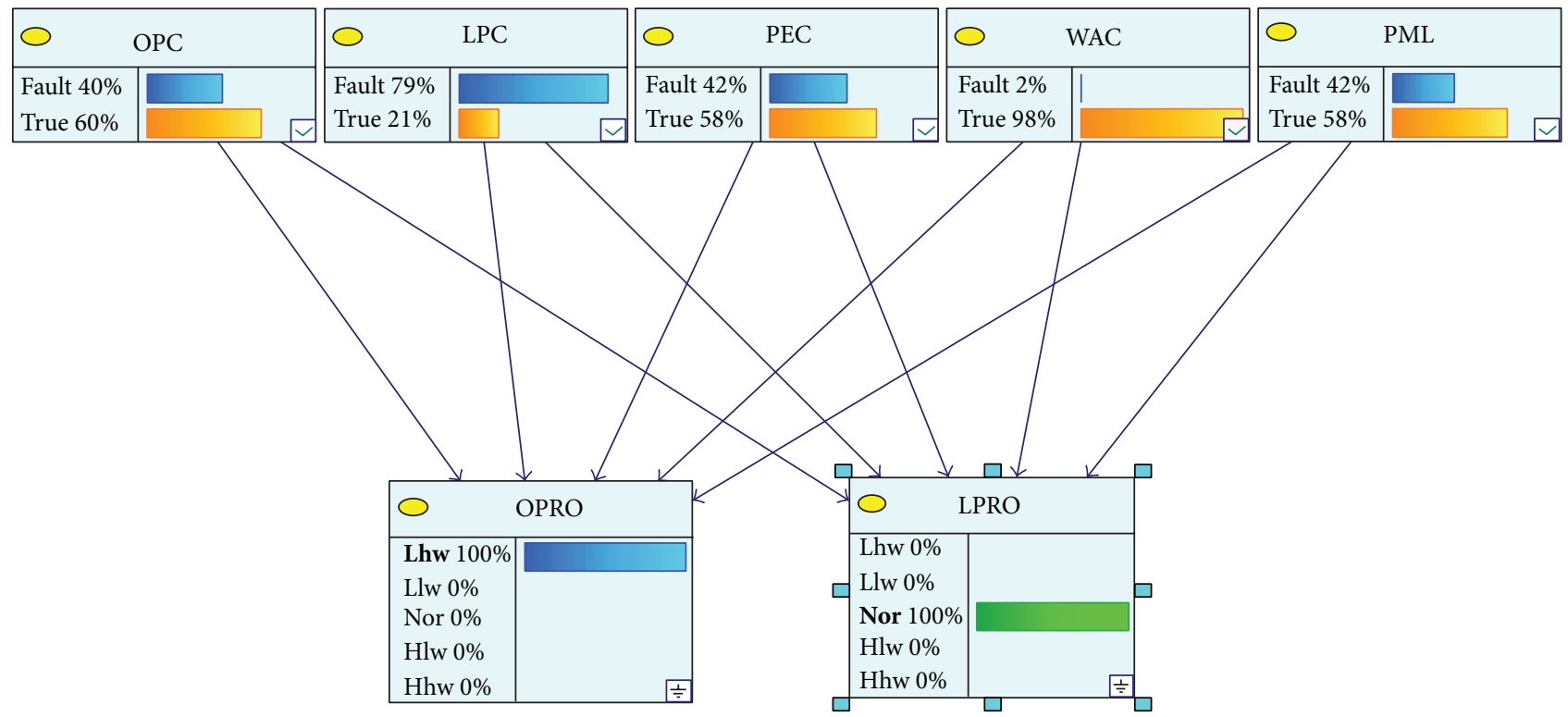

FIGURE 5: The posterior probability distribution under the evidence of OPRO heavy warning and LPRO Normal.

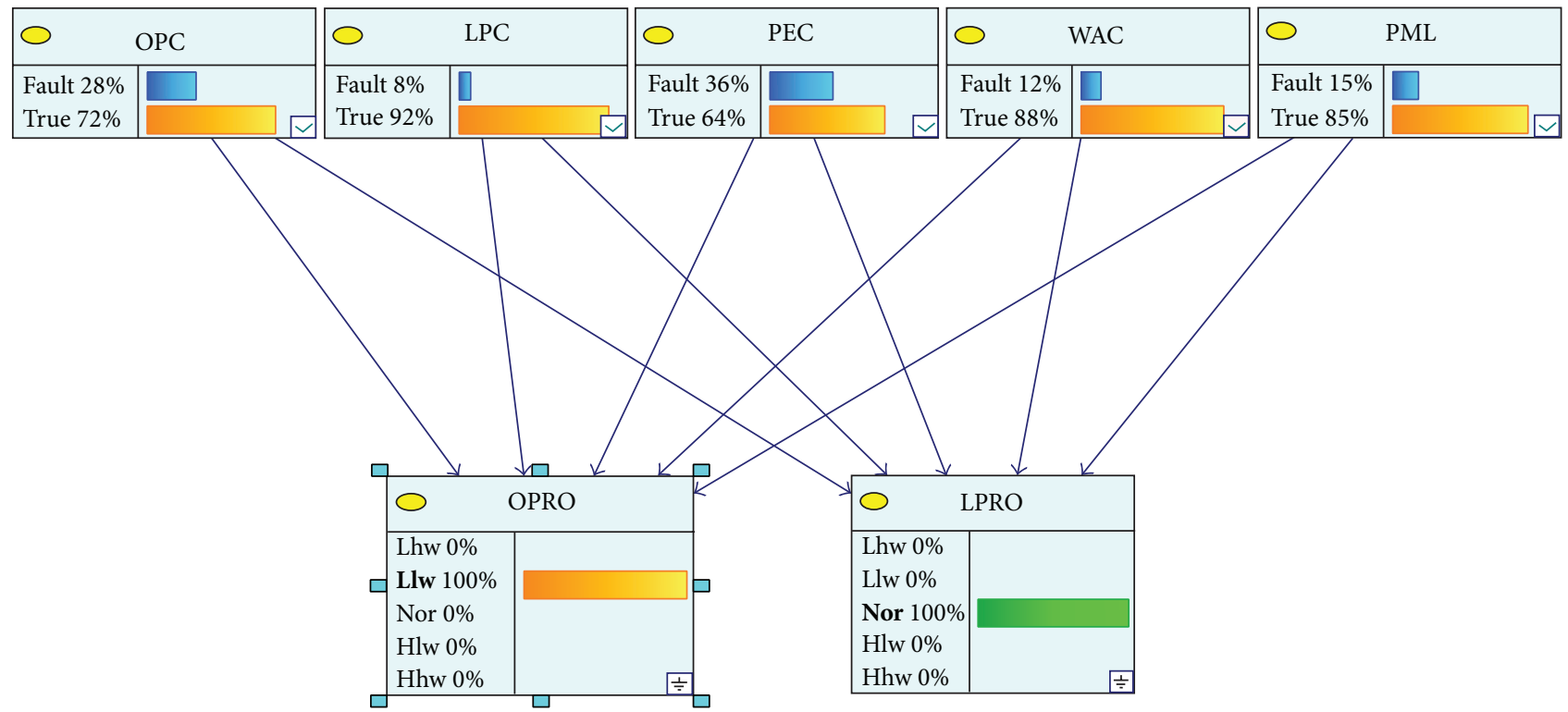

FIGURE 6: The posterior probability distribution under the evidence of OPRO light warning and LPRO Normal.

of PEC fault are 36\%. Reasoning results are consistent with the reality.

Figure 7 shows that the most likely risk point which causes such evidence is WAC, for the posterior probabilities of WAC fault are $69 \%$. Reasoning results correspond to the actual situation.

4.3.3. The Analysis of the Results. It can be drawn from the three-group reasoning results above that the most influential indexes are WAC and LPC in the high water-cut period of oilfield development. They contact closely with the amount of water injected, which are positively correlated. In other words, we can increase the water injection to raise the oil production in the later stages of oilfield development. The inference result is very consistent with the engineering practice.

\section{Conclusion}

Based on the studies for oilfield development risk forecasting and early warning of Bayesian network, we integrated the neural networks and Bayesian networks successfully to 


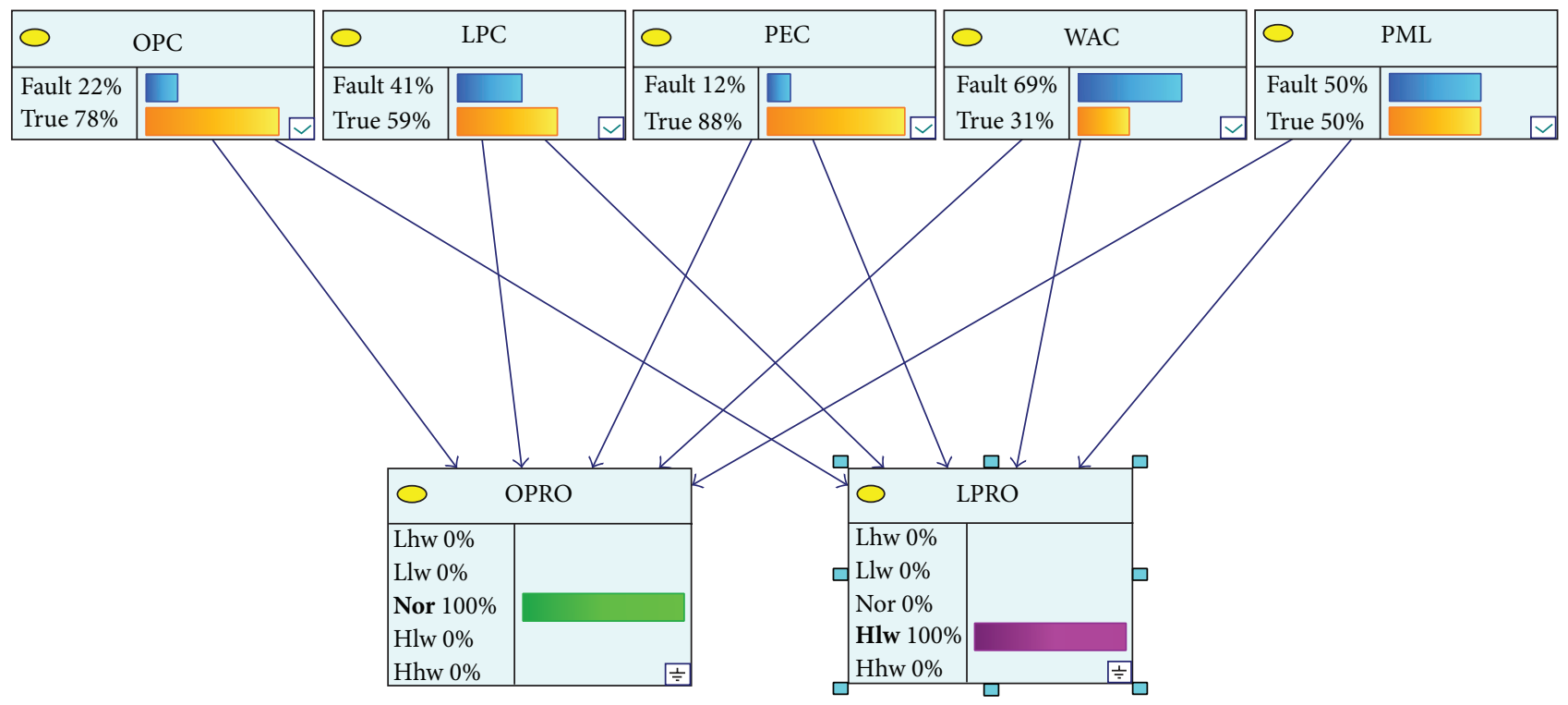

FIGURE 7: The posterior probability distribution under the evidence of OPRO Normal and LPRO light warning.

present a new method of forecasting and early warning of oilfield development risk. This method uses neural network to predict the warning situation indexes of oilfield development risk, uses " $3 \sigma$ " rule to determine the interval of risk warning degree, and uses Bayesian network to diagnose the reasons causing the risk. The case study shows that the new method proposed can provide help and guidance for discovering and controlling oilfield development risk. The revised $\mathrm{BN}$ method proposed by us is different from the existing $\mathrm{BN}$ method revised by fault trees or event trees or other methods. In the existing revised BN methods, the structure model and parameter of the Bayesian network are determined largely by fault trees or event trees or other methods, and the main purpose of the $\mathrm{BN}$ is to calculate posterior probability distribution of node (risk) after joining the evidence, while in our revised BN method, neural network is first used to predict warning situation indexes. Subsequently, ascertain the degree of the predicted value, and the degree value will be as input evidence of $\mathrm{BN}$ whose structure model is built by historical experience and parameter model based on the sample data to calculate the posterior probability distribution of node under the evidence. The future works may be to expand our research by introducing dynamic BN or to develop the software on oilfield development risk forecasting and early warning.

\section{Conflict of Interests}

The authors declare that there is no conflict of interests regarding the publication of this paper.

\section{Acknowledgment}

The authors are grateful for financial support from scientific research innovation team fund of Southwest Petroleum
University (2013XJZT004): “The Optimization Theory and Control."

\section{References}

[1] F. Khan, S. Rathnayaka, and S. Ahmed, "Methods and models in process safety and risk management: past, present and future," Process Safety and Environmental Protection, vol. 98, pp. 116-147, 2015.

[2] F. Khan, S. Ahmed, M. Yang et al., "Safety challenges in harsh environments: lessons learned," Process Safety Progress, vol. 34, no. 2, pp. 191-195, 2015.

[3] M. Musharraf, D. Bradbury-Squires, F. Khan, B. Veitch, S. Mackinnon, and S. Imtiaz, "A virtual experimental technique for data collection for a Bayesian network approach to human reliability analysis," Reliability Engineering and System Safety, vol. 132, pp. 1-8, 2014.

[4] N. Khakzad, F. Khan, and P. Amyotte, "Quantitative risk analysis of offshore drilling operations: a Bayesian approach," Safety Science, vol. 57, pp. 108-117, 2013.

[5] M. Abimbola, F. Khan, N. Khakzad et al., "Safety and risk analysis of managed pressure drilling operation using Bayesian network," Safety Science, vol. 76, pp. 133-144, 2015.

[6] J. E. Vinnem, R. Bye, B. A. Gran et al., "Risk modelling of maintenance work on major process equipment on offshore petroleum installations," Journal of Loss Prevention in the Process Industries, vol. 25, no. 2, pp. 274-292, 2012.

[7] J. Bhandari, R. Abbassi, V. Garaniya, and F. Khan, "Risk analysis of deepwater drilling operations using Bayesian network," Journal of Loss Prevention in the Process Industries, vol. 38, pp. 11-23, 2015.

[8] N. Khakzad, F. Khan, and P. Amyotte, "Dynamic safety analysis of process systems by mapping bow-tie into Bayesian network," Process Safety and Environmental Protection, vol. 91, no. 1-2, pp. 46-53, 2013. 
[9] H. Yu, F. Khan, and V. Garaniya, "Modified independent component analysis and bayesian network-based two-stage fault diagnosis of process operations," Industrial \& Engineering Chemistry Research, vol. 54, no. 10, pp. 2724-2742, 2015.

[10] Z. Yuan, N. Khakzad, F. Khan, and P. Amyotte, "Risk analysis of dust explosion scenarios using Bayesian networks," Risk Analysis, vol. 35, no. 2, pp. 278-291, 2015.

[11] N. Khakzad, "Application of dynamic Bayesian network to risk analysis of domino effects in chemical infrastructures," Reliability Engineering \& System Safety, vol. 138, pp. 263-272, 2015.

[12] N. Khakzad, S. Khakzad, and F. Khan, "Probabilistic risk assessment of major accidents: application to offshore blowouts in the Gulf of Mexico," Natural Hazards, vol. 74, no. 3, pp. 17591771, 2014.

[13] A.-A. Baksh, F. Khan, V. Gadag, and R. Ferdous, "Network based approach for predictive accident modelling," Safety Science, vol. 80, pp. 274-287, 2015.

[14] M. Kalantarnia, F. Khan, and K. Hawboldt, "Dynamic risk assessment using failure assessment and Bayesian theory," Journal of Loss Prevention in the Process Industries, vol. 22, no. 5, pp. 600-606, 2009.

[15] X. Yang, W. J. Rogers, and M. S. Mannan, "Uncertainty reduction for improved mishap probability prediction: application to level control of distillation unit," Journal of Loss Prevention in the Process Industries, vol. 23, no. 1, pp. 149-156, 2010.

[16] C. E. Alaneme and A. C. Igboanugo, "A fuzzy-logic theoretic approach to modelling marginal oilfield risks," Research Journal of Applied Sciences, Engineering and Technology, vol. 6, no. 4, pp. 557-567, 2013.

[17] S. M. M. Lavasani, Z. Yang, J. Finlay, and J. Wang, "Fuzzy risk assessment of oil and gas offshore wells," Process Safety and Environmental Protection, vol. 89, no. 5, pp. 277-294, 2011.

[18] D. J. C. Mackay, "Probable networks and plausible predictionsa review of practical bayesian methods for supervised neural networks," Network: Computation in Neural Systems, vol. 6, no. 3, pp. 469-505, 1995.

[19] R. M. Neal, Bayesian Learning for Neural Networks, Springer, New York, NY, USA, 1996.

[20] J. F. G. Freitas, Bayesian methods for neural networks [Ph.D. thesis], Trinity College University of Cambridge and Cambridge University Engineering Department, Cambridge, UK, 2000.

[21] N. Chitsazan, A. A. Nadiri, and F. T.-C. Tsai, "Prediction and structural uncertainty analyses of artificial neuralnetworks using hierarchical Bayesian model averaging," Journal of Hydrology, vol. 528, pp. 52-62, 2015.

[22] J. L. Ticknor, "A Bayesian regularized artificial neural network for stock market forecasting," Expert Systems with Applications, vol. 40, no. 14, pp. 5501-5506, 2013.

[23] C. Maschio and D. J. Schiozer, "Bayesian history matching using artificial neural network and Markov Chain Monte Carlo," Journal of Petroleum Science and Engineering, vol. 123, pp. 62-71, 2014.

[24] W. Mingda, C. Guoming, F. Jianmin, and L. Weijun, "Safety analysis approach of MFM-HAZOP and its application in the dehydration system of oilfield united station," Procedia Engineering, vol. 43, pp. 437-442, 2012.

[25] R. Sadiq, T. Husain, B. Veitch, and N. Bose, "Risk-based decision-making for drilling waste discharges using a fuzzy synthetic evaluation technique," Ocean Engineering, vol. 31, no. 16, pp. 1929-1953, 2004.
[26] J. Kang, W. Liang, L. Zhang et al., "A new risk evaluation method for oil storage tank zones based on the theory of two types of hazards," Journal of Loss Prevention in the Process Industries, vol. 29, no. 1, pp. 267-276, 2014.

[27] J. E. Horner, J. W. Castle, and J. H. Rodgers Jr., "A risk assessment approach to identifying constituents in oilfield produced water for treatment prior to beneficial use," Ecotoxicology and Environmental Safety, vol. 74, no. 4, pp. 989-999, 2011.

[28] D. Baca and K. Petersen, "Countermeasure graphs for software security risk assessment: an action research," Journal of Systems and Software, vol. 86, no. 9, pp. 2411-2428, 2013.

[29] T. Wang, P. Wang, J. Meng et al., "A review of sources, multimedia distribution and health risks of perfluoroalkyl acids (PFAAs) in China," Chemosphere, vol. 129, pp. 87-99, 2015.

[30] D. Kim, S. Kim, and T. Yoo, "Osteoarthritis and cartilage," in Proceedings of the World Congress of the Osteoarthritis Research Society International (OARSI '14), p. S379, Elsevier Science, Paris, France, April 2014.

[31] R. S. Targino, G. W. Peters, and P. V. Shevchenko, "Sequential Monte Carlo samplers for capital allocation under copuladependent risk models," Insurance: Mathematics and Economics, vol. 61, pp. 206-226, 2015.

[32] M. Hofmann, R. Lux, and H. R. Schultz, "Constructing a framework for risk analyses of climate change effects on the water budget of differently sloped vineyards with a numeric simulation using the Monte Carlo method coupled to a water balance model," Frontiers in Plant Science, vol. 5, article 645, 2014.

[33] E. J. D. S. Pereira, J. T. Pinho, M. A. B. Galhardo, and W. N. Macêdo, "Methodology of risk analysis by Monte Carlo Method applied to power generation with renewable energy," Renewable Energy, vol. 69, pp. 347-355, 2014.

[34] L. Han, Y. Song, L. Duan, and P. Yuan, "Risk assessment methodology for Shenyang Chemical Industrial Park based on fuzzy comprehensive evaluation," Environmental Earth Sciences, vol. 73, no. 9, pp. 5185-5192, 2015.

[35] Y. Wang, J. Zhang, E. Guo, and Z. Sun, "Fuzzy comprehensive evaluation-based disaster risk assessment of desertification in Horqin Sand Land, China," International Journal of Environmental Research and Public Health, vol. 12, no. 2, pp. 1703-1725, 2015.

[36] A. C. Constantinou, N. E. Fenton, and M. Neil, "Profiting from an inefficient association football gambling market: prediction, risk and uncertainty using Bayesian networks," KnowledgeBased Systems, vol. 50, pp. 60-86, 2013.

[37] E. Sharifahmadian and S. Latifi, "Water environment risk prediction using Bayesian network," in Proceedings of the IEEE SoutheastCon, pp. 1-5, Jacksonville, Fla, USA, April 2013.

[38] Z. Zhao, Z. Liu, J. Xu, and H. Jiang, "Analysis and prediction of Block detection indexes," in Early Warning Dynamic System in the Oilfield Development, Z. Zhiyong, Ed., Petroleum Industry Press, Beijing, China, 2010.

[39] Y. Zhong, Research on the Dynamic Prediction Methods for Extra High Water Cut Oilfield Development Planning, Southwest Petroleum University, 2008.

[40] J. Botzheim and P. Földesi, "Novel calculation of fuzzy exponent in the sigmoid functions for fuzzy neural networks," Neurocomputing, vol. 129, pp. 458-466, 2014.

[41] D. Xia, L. Kong, Y. Hu, and P. Ni, "Silicon microgyroscope temperature prediction and control system based on BP neural network and fuzzy-PID control method," Measurement Science \& Technology, vol. 26, no. 2, Article ID 025101, 2015. 
[42] R. Lehmann, "3 Sigma-rule for outlier detection from the viewpoint of geodetic adjustment," Journal of Surveying Engineering, vol. 139, no. 4, pp. 157-165, 2013.

[43] D. Fu, "Research on the learning algorithm of Bayesian Network," Journal of Daqing Normal University, vol. 31, no. 3, pp. 36-38, 2011.

[44] M. R. Martins, A. M. Schleder, and E. L. Droguett, "A methodology for risk analysis based on hybrid bayesian networks: application to the regasification system of liquefied natural gas onboard a floating storage and regasification unit," Risk Analysis, vol. 34, no. 12, pp. 2098-2120, 2014.

[45] X. Li, "Research on learning Bayesian network parameters," Journal of Shenyang Agricultural University, vol. 38, no. 1, pp. 125-128, 2007. 


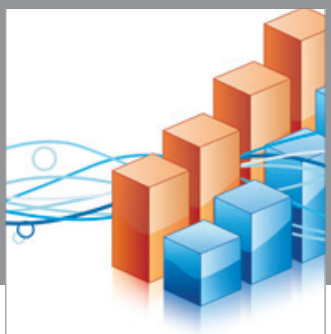

Advances in

Operations Research

vatem alat4

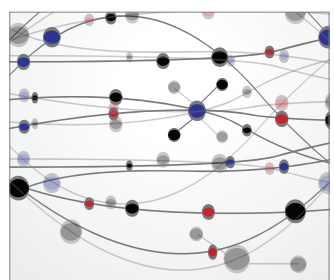

\section{The Scientific} World Journal
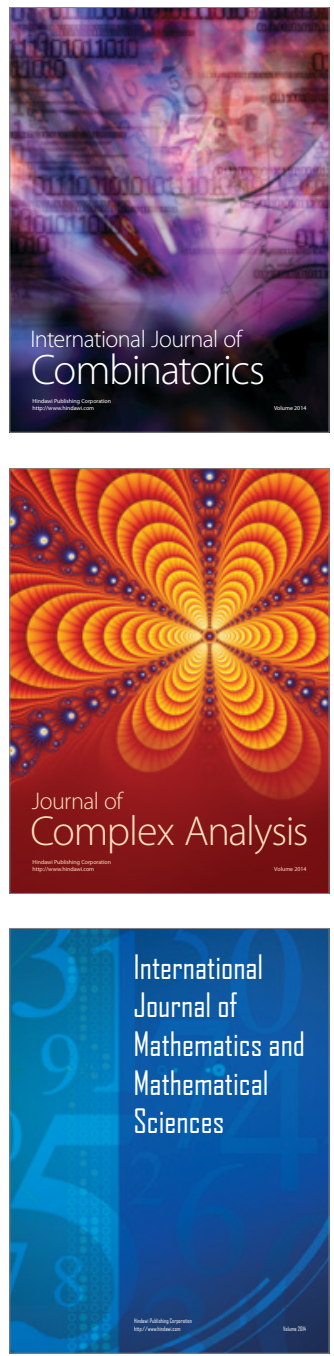
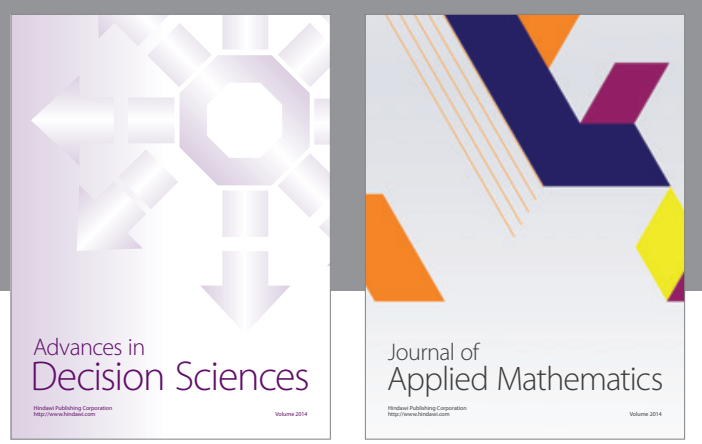

Algebra

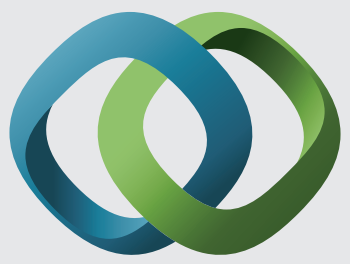

\section{Hindawi}

Submit your manuscripts at

http://www.hindawi.com
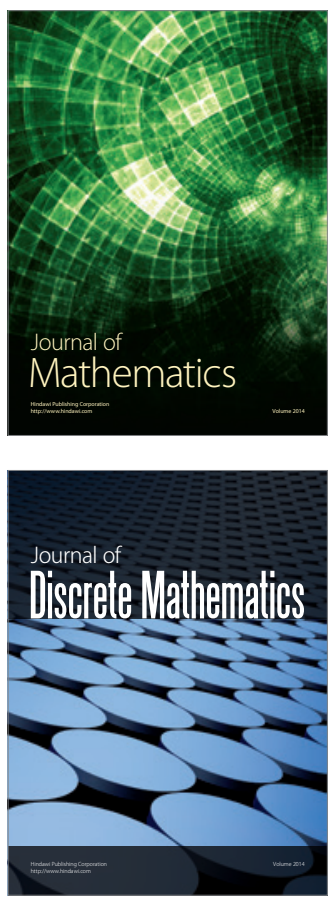

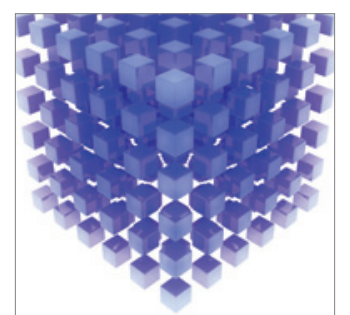

Mathematical Problems in Engineering
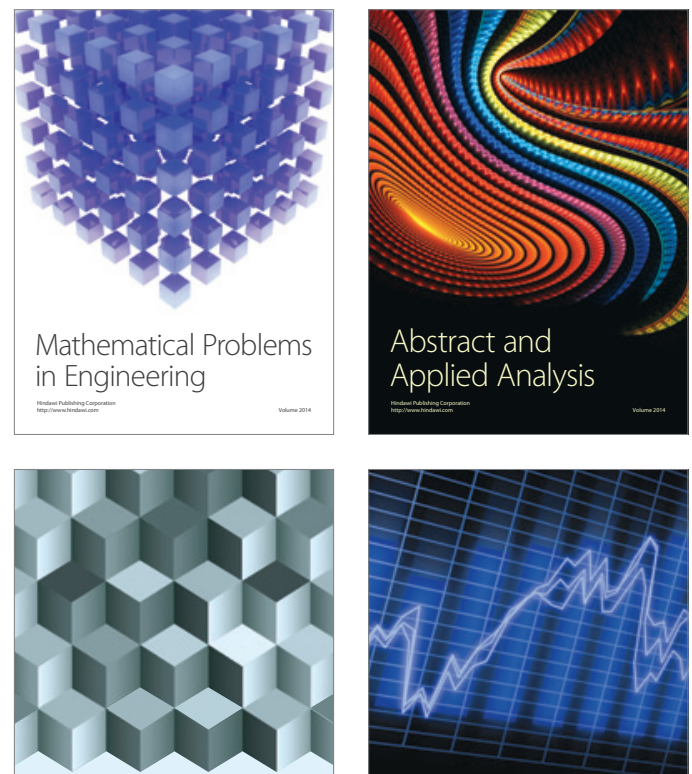

Journal of

Function Spaces

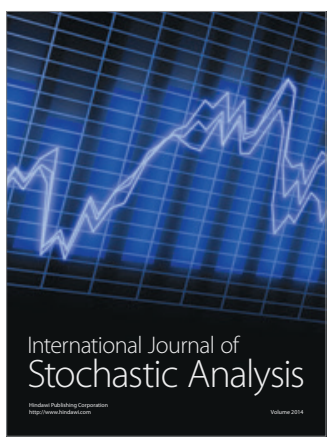

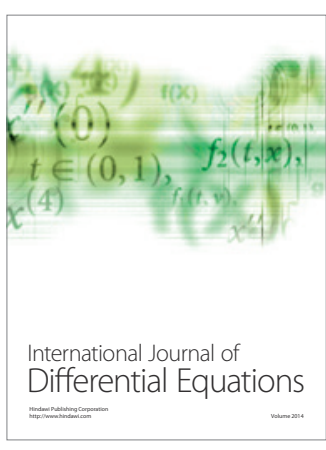
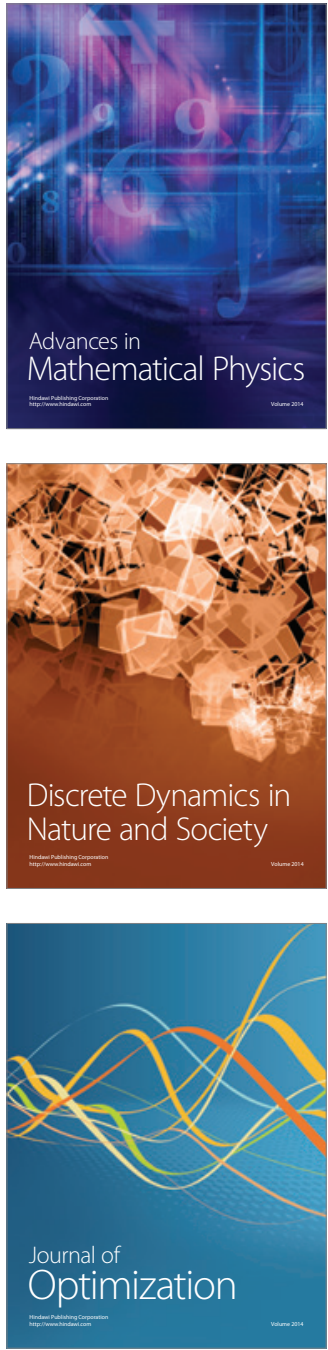